\title{
A weak lensing analysis of a STIS dark-lens candidate ${ }^{\star}$
}

\author{
T. Erben ${ }^{1}$, J. M. Miralles ${ }^{1}$, D. Clowe $^{1}$, M. Schirmer ${ }^{1}$, P. Schneider ${ }^{1}$, W. Freudling ${ }^{2}$, \\ N. Pirzkal ${ }^{2}$, R. A. E. Fosbury ${ }^{2}$, and B. Jain ${ }^{3}$ \\ ${ }^{1}$ Institut für Astrophysik und Extraterrestrische Forschung (IAEF), Universität Bonn, Auf dem Hügel 71, 53121 Bonn, \\ Germany \\ 2 ST-ECF, European Southern Observatory, Karl-Schwarzschild Str. 2, 85741 Garching, Germany \\ 3 Department of Physics and Astronomy, University of Pennsylvania, Philadelphia, PA 19104, USA
}

Received 25 April 2003 / Accepted 31 July 2003

\begin{abstract}
We perform a weak lensing analysis on a previously reported dark-lens candidate on STIS Parallel data (Miralles et al. 2002). New VLT-data indicate that the reported signal originates from a small number of galaxies tangentially aligned towards the center of the STIS field but no signature for an extended mass distribution is found. We argue that we should be able to detect a massive cluster $\left(M \geq 3.2 \times 10^{14} M_{\odot}\right)$ through its lensing signal up to a redshift of $z \approx 0.6$ with our data. Also the double image hypothesis of two galaxies with very similar morphologies in the STIS data is ruled out with colour information.
\end{abstract}

Key words. galaxies: clusters: general

\section{Introduction}

In the last 5 years, the weak lensing technique has provided us with important insights into the dark matter distribution of known low- and high-redshift galaxy clusters (see e.g. Clowe et al. 2000; Hoekstra et al. 1998, 2000). One of the advantages of this technique over others is that it does not rely on the relation between the light emission of an object and its mass, but as Kaiser \& Squires (1993) showed, the surface mass density of massive objects can be reconstructed from the distortion induced by its tidal gravitational field on background galaxies.

We can turn around the argument and use this technique to blindly search for new, hitherto unknown mass concentrations. So far, 9 candidates for such mass concentrations detected by weak lensing techniques have been reported in the literature (Erben et al. 2000; Umetsu \& Futamase 2000; Mellier et al. 2000; Wittman et al. 2001; Miralles et al. 2002; Wittman et al. 2002; Dahle et al. 2003). Five of them (Wittman et al. 2001, 2002; Mellier et al. 2000, and two of the three candidates in Dahle et al. 2003) have been confirmed as galaxy clusters. The other four candidates lack optical counterparts and are thus potentially dark clusters with an unusually high $M / L$ ratio. As the firm confirmation of only one such dark cluster would already have severe consequences for current cluster formation scenarios and for the nature of dark matter (Verde et al. 2000; Weinberg \& Kamionkowski 2002), careful and independent analyses with follow-up observations have to be done on such candidates to either reject or strengthen the cluster hypothesis

Send offprint requests to: T. Erben,

e-mail: terben@astro.uni-bonn.de

^ Based on observations with FORS2@VLT operated by ESO. and to reveal possible, hitherto unknown systematics in weak lensing analyses.

In this paper we present a weak lensing analysis of groundbased images of the lens candidate detected in an HST/STIS parallel field (Miralles et al. 2002, MEH henceforth). The highresolution STIS image yielded the strong visual impression of a gravitational lens system. It showed several extended objects aligned tangentially to the field centre, as well as a pair of galaxies with similar morphologies and surface brightness which seemed to be a potential strong lensing, double-image candidate, thus indicating lensing by a massive structure. We show in this work that, despite the first indications, the observed object configurations in this field most probably do not originate from gravitational lensing. The paper is organised as follows: First we present the new VLT-observations. Thereafter, we make theoretical predictions about the detectability of a massive galaxy cluster with our data. We continue by presenting our weak lensing analysis and finish with our conclusions.

\section{The data}

The VLT-data for the current work were obtained in an ESO Director's Discretionary Time Proposal (269.A-5064). The set was observed in the nights of 05-06/10/2002 with the newly installed FORS2@UT4 camera having a field-of-view of approximately $7^{\prime} \times 7^{\prime}$. The camera consists of two $2 \mathrm{~K} \times 4 \mathrm{~K}$ CCDs with a gap of about $5^{\prime \prime}$ oriented in the West-East direction. We obtained $3120 \mathrm{~s} I$-band observations in unbinned mode (pixel scale $0{ }^{\prime} 126$ ) as our primary weak lensing science band. Furthermore, we obtained exposures of $1560 \mathrm{~s}$ in $V$ and $6380 \mathrm{~s}$ 
in $B$ in the standard binned mode (pixel scale 0.252 ) for detecting a possible red-cluster sequence and to study the stronglensing hypothesis of a double-image candidate. The data in each band were centered on the position of the STIS field and we applied a dithering pattern between each individual exposure in order to minimise the effects of the gap and other defects (hot pixels, bad columns) of the detectors in the final coadded images.

The data processing was carried out with a pipeline developed specifically for the reduction of multi-chip cameras that is described in Schirmer et al. (2003). In the following we only describe the astrometric calibration, whose accuracy is essential for weak lensing studies, in some more detail.

After preprocessing, the individual images were astrometrically calibrated by comparing object positions with those from the USNO-A2 astrometric catalogue (Monet et al. 1996) having 80 sources in our field. We used Mario Radovich's ASTROMETRIX $^{1}$ to fit image distortions by a third-order, two-dimensional polynomial for every individual chip. Hereby, distances from objects to the standard star catalogue and to overlap sources in other chips are minimised in the $\chi^{2}$ sense. This procedure gave us positional rms residuals of 0 !' 15-0!' 28 (this is approximately the positional accuracy of the USNO-A2 catalogue) for the standard stars and 0.'005-0.'01 for the overlap sources in the $I$ band. Hence, we are able to align the images with an internal accuracy of $1 / 20-1 / 10$ of a pixel; see also Fig. 1. The final coaddition was performed with the drizzle package (Fruchter \& Hook 2002). All the data have been obtained in photometric conditions and photometric zeropoints were provided by ESO. The measured seeing in the final coadded $I$ image is about 0.7 . A first object catalogue was created with SExtractor (Bertin \& Arnouts 1996), where we considered all detections with 5 contiguous pixels with $2.5 \sigma$ over the sky background noise. With these parameters we reached a completeness level of $I \approx 24$ in our primary science frame. All magnitudes quoted in this paper are in the Vega system.

\section{Detectability of massive structures in our data}

In the following we use standard lensing notation. For a broader introduction to the topic, see for instance Bartelmann \& Schneider (2001). To estimate the detectability of a galaxy cluster in our current data, we use the $M_{\text {ap }}$ statistic introduced by Schneider (1996). It is defined as

$M_{\mathrm{ap}}=\int_{0}^{\theta} \mathrm{d}^{2} \boldsymbol{\vartheta} \kappa\left(\boldsymbol{\vartheta}, z_{\mathrm{d}}, z_{\mathrm{s}}\right) U(\vartheta)$

where $\int_{0}^{\theta} \mathrm{d} \vartheta U(\vartheta)=0$, i.e. $U(\vartheta)$ is a compensated filter and $z_{\mathrm{d}}$, $z_{\mathrm{s}}$ are the redshifts of lens and source, respectively. Throughout this work we use $U(\vartheta)=u(\vartheta / \theta) / \theta^{2} ; u(\rho)=(9 / \pi)\left(1-\rho^{2}\right)$ $\left(1 / 3-\rho^{2}\right) . M_{\text {ap }}$ represents a filtered integral over the surface mass distribution $\kappa$ and can be related to the tangential shear $\gamma_{\mathrm{t}}$ via

$M_{\text {ap }}=\int_{0}^{\theta} \mathrm{d}^{2} \boldsymbol{\vartheta} \gamma_{\mathrm{t}}(\boldsymbol{\vartheta}) Q(\vartheta)$

\footnotetext{
${ }^{1}$ See http://www.na.astro.it/ radovich/wifix.htm
}

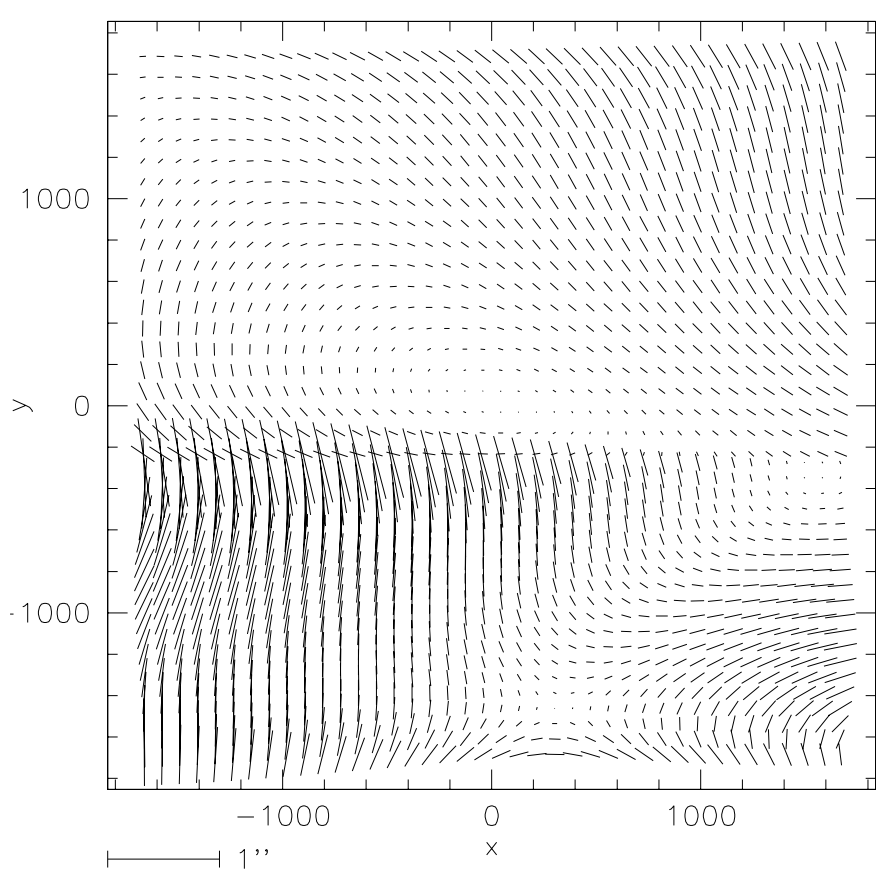

Fig. 1. We illustrate the distortion map from the FORS2 instrument. The sticks represent the positional difference of a simple shift to match sources in the field with the USNO-A2 catalogue compared with a full third order astrometric solution. The maximum difference is about 6.19 pixel ( 0 '.78). Over a maximum distance of about 2500 pixels from the optical axis, the distortion is about $0.247 \%$ which is better than the specification ( $0.3 \%$; see also FORS1+2 User Manual). The origin of the plot is at the nominal optical axis. We note that distortions in the lower chip are significantly higher than in the upper one. Correcting for the distortion gives us between $1 / 20-1 / 10$ pixel internal astrometric accuarcy for overlap objects.

with

$Q(\vartheta)=\frac{2}{\vartheta^{2}} \int_{0}^{\vartheta} \mathrm{d} \vartheta^{\prime} \vartheta^{\prime} U\left(\vartheta^{\prime}\right)-U(\vartheta)$.

For the application to data, it is straightforward to construct an unbiased estimate $M_{\text {ap }}^{\prime}$ for the integral by a discrete sum over observed galaxy ellipticities $\epsilon_{\mathrm{t}}$ and considering the coordinate origin being at the center of the aperture:

$M_{\mathrm{ap}}^{\prime}=\frac{\pi \theta^{2}}{N} \sum_{i} \epsilon_{\mathrm{t}}\left(\boldsymbol{\vartheta}_{i}\right) Q\left(\vartheta_{i}\right)$

As $M_{\text {ap }}$ is a scalar quantity, expectation values for the noise $\sigma_{\text {Map }}$ of a measurement are easily evaluated:

$\sigma_{\text {Map }}^{2}=\frac{\pi \sigma_{\epsilon}^{2}}{n} \int_{0}^{\theta} \mathrm{d} \vartheta \vartheta Q^{2}(\vartheta) \rightarrow \frac{\sigma_{\epsilon}^{2}}{2 n^{2}} \sum_{i} Q^{2}\left(\vartheta_{i}\right)$,

where $\sigma_{\epsilon}$ is the ellipticity dispersion and $n$ the number density of galaxies.

For obtaining predictions for expected signal-to-noise $(S / N)$ ratios for halos with a mass $M$ at a redshift $z_{\mathrm{d}}$, we have to specify a mass model $\kappa\left(\boldsymbol{\vartheta}, z_{\mathrm{d}}, z_{\mathrm{s}}\right)$ and a distribution for the source redshifts $p\left(z_{\mathrm{s}}\right)$. The expected signal $S$ is then given by

$S=\int_{0}^{\theta} \mathrm{d}^{2} \boldsymbol{\vartheta} \int_{z_{\mathrm{d}}}^{\infty} \mathrm{d} z_{\mathrm{s}} \kappa\left(\boldsymbol{\vartheta}, z_{\mathrm{d}}, z_{\mathrm{s}}\right) U(\vartheta) p\left(z_{\mathrm{s}}\right)$. 


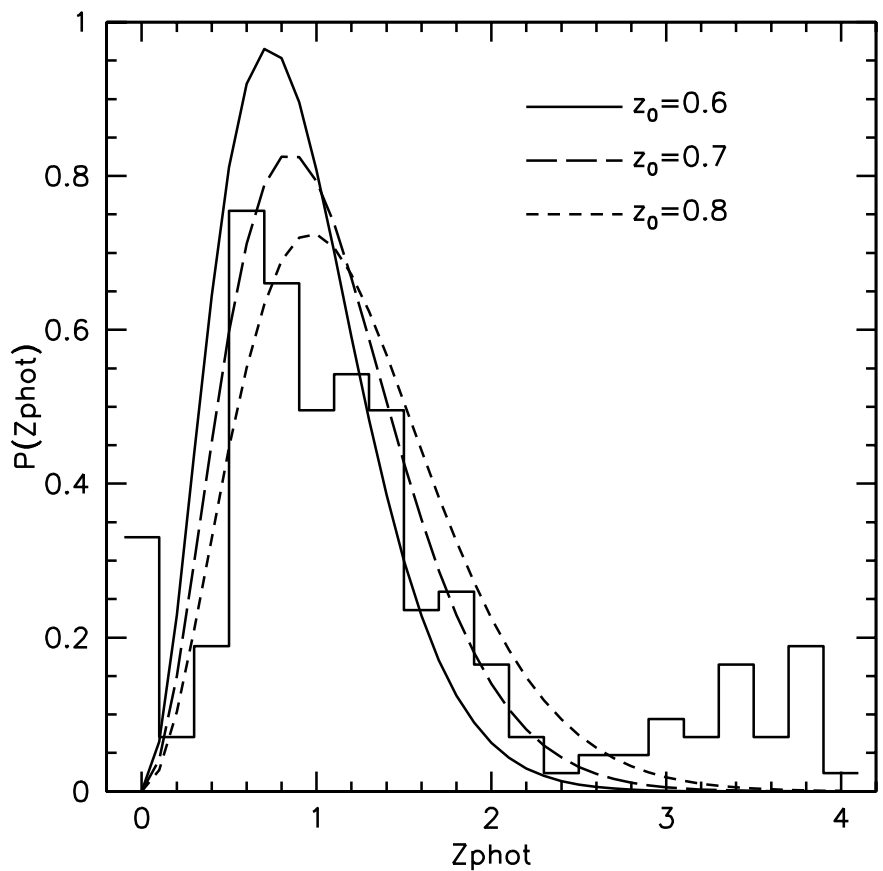

Fig. 2. Shown is the normalised photometric redshift distribution $P($ Zphot $)$ around the galaxy cluster RXJ $1347-1145(z=0.451)$ in UBVRI photometry. It was estimated with hyperz (see Bolzonella et al. 2000, and http://webast.ast.obs-mip.fr/hyperz). We show only objects with a good redshift model fit $P\left(\chi^{2}\right)>90.0 \%$ and cut away all galaxies having a probability of higher than $60 \%$ to be at the cluster redshift of $z=0.451$. Although we discovered several high redshift candidates in the field, the large number of objects having a formal photometric redshift of larger than 3 is probably an artefact due to missing infrared information. The three curves are model predictions according to Eq. (7) with $z_{0}=0.6$ (solid curve); $z_{0}=0.7$ (long-dashed curve) and $z_{0}=0.8$ (short-dashed curve).

The noise is given by $\sigma_{\text {Map }}$ in (5). For the mass profile we consider the universal density profile proposed by Navarro et al. (1996). The details, how the surface mass density $\kappa\left(\boldsymbol{\vartheta}, z_{\mathrm{d}}, z_{\mathrm{s}}\right)$ is obtained for this profile when fixing the halo mass $M$, are given in (Bartelmann 1996; Kruse \& Schneider 1999) and will not be repeated here. For the source redshifts we consider the normalised distribution:

$p\left(z_{\mathrm{s}}\right)=\frac{3}{2 z_{0}}\left(\frac{z_{\mathrm{s}}}{z_{0}}\right)^{2} \exp \left[-\left(\frac{z_{\mathrm{s}}}{z_{0}}\right)^{1.5}\right]$

which was proposed in Brainerd et al. (1996). To fix $z_{0}$, we considered the redshift distribution from $U B V R I$ photometry around the galaxy cluster RXJ1347-1145. The data for this cluster will be described elsewhere (Erben et al., in preparation). They have been obtained with FORS1 around the cluster center (field-of-view $3^{\prime} \times 3^{\prime}$ ). As for the current data set, the $I$ band of these observations is used as the primary science band for a weak lensing study of the cluster. As the two observations reach about the same depth $(I \approx 24)$, we expect that the source galaxies trace comparable redshift distributions. The redshift distribution for the cluster field is shown in Fig. 2. We estimate from this figure that $z_{0}=0.7$ (and thus $\left\langle z_{\mathrm{s}}\right\rangle=1.05$ ) provides a fair redshift description for the source distribution in our current data. As the lens magnification effect of the massive cluster

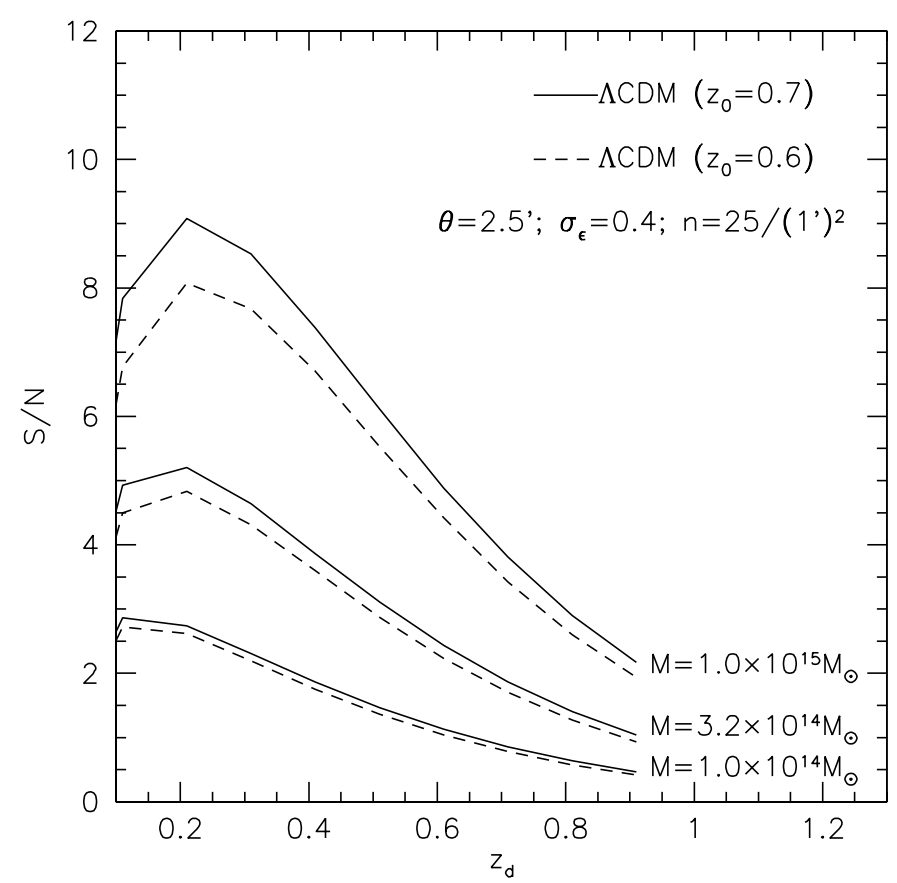

Fig. 3. Shown are $S / N$ predictions for the detectability of massive structures in our VLT data. We plot predictions for a $\Lambda$ CDM cosmology $\left(\Omega_{0}=0.3 ; \Omega_{\Lambda}=0.7 ; \sigma_{8}=0.9 ; \Gamma=0.25\right)$ and two different redshift distributions (see Eq. (7)). We assumed a smoothing scale of $\theta=2.5$ (Kruse \& Schneider 1999 showed that for a smoothing scale of about $2^{\prime}, M_{\text {ap }}$ gives the maximum significance for halos following a NFW profile), a number density of 25 galaxies per sq. arcminute and $\sigma_{\epsilon}=0.4$ for the width of the ellipticity distribution (measured to be $\sigma_{\epsilon}=0.42$ in our data). The results do not change significantly if we consider an EdS universe $\left(\Omega_{0}=1.0 ; \Omega_{\Lambda}=0.0 ; \sigma_{8}=0.6 ; \Gamma=0.25\right)$ instead.

might significantly push the redshift distribution to higher values we also investigate the case of $z_{0}=0.6 ;\left\langle z_{\mathrm{s}}\right\rangle=0.9$.

Figure 3 shows our predictions for $S / N$ ratios from the described model. We conclude that we are insensitive to structures below $10^{14} M_{\odot}$ and can marginally detect, at the $3 \sigma$ level, clusters of about $3 \times 10^{14} M_{\odot}$ up to a redshift of $z \approx 0.6$.

\section{Weak lensing analysis of the VLT-data}

To cross-check our weak lensing results, we performed two independent analyses of the data.

\section{Analysis 1:}

Starting from the initial SExtractor catalogue we determined, for all the objects, the quantities necessary to obtain shear estimates according to the KSB (Kaiser et al. 1995) algorithm. To correct all galaxies for PSF anisotropy and PSF smearing effects we closely follow the procedures described in Erben et al. (2001). There we introduced a weighting scheme for individual galaxies based on the ellipticity distribution of the corrected ellipticities. Since in the current paper we were looking for a potential strong lensing cluster, high ellipticities could be caused by lensing and do not necessarily reflect the true noise properties. Hence, we did not use this weighting scheme for the current work but conservatively rejected all 


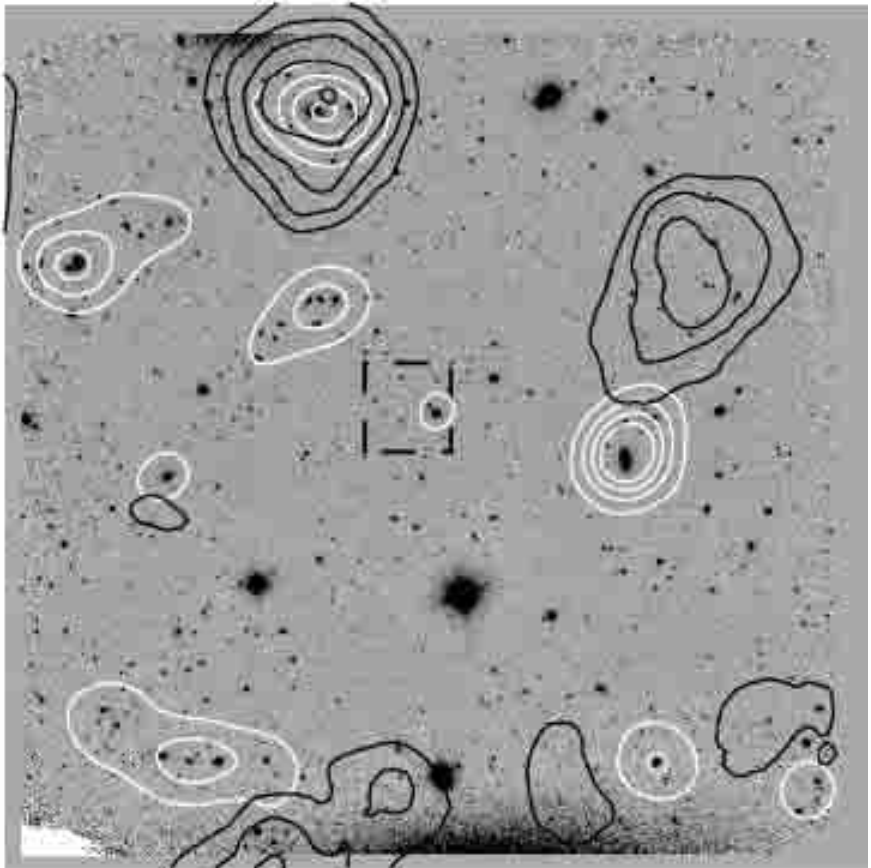

Fig. 4. Shown is the light distribution (white contours) and $M_{\text {ap }}$ significance contours (black contours) in our VLT field. The light distribution contains all galaxies also used for the weak lensing analysis. It is estimated on a regular grid, where each gridpoint contains the total flux within $1^{\prime}$ weighted by a Gaussian with a width of 22 '. 5 . The light contours enclose regions where the light is $3,6,9$ and $12 \sigma$ above the mean light. The $M_{\text {ap }}$ contours display mass significance of 1.0, 1.5, 2.0, 2.5 and 3.0 $\sigma$ with a smoothing scale of 2'.5. The dashed rectangle marks the area of the original HST/STIS observation. We see that no significant detection is found at the position of our dark lens candidate.

galaxies having a corrected ellipticity larger than 0.8 . The final catalogue for the lensing analysis contains 1200 objects, i.e. around 25 per square arcmin.

Figures 4 and 5 show the $M_{\text {ap }}$ statistics result for our data. As can be seen we do not recover any significant mass concentration at the position of our original lens candidate when the filter scale becomes larger than the size of the original STIS image. We can recover a $3 \sigma$ peak with a filter scale of $30^{\prime \prime}$ which is consistent with the $2.5 \sigma$ detection reported on the original STIS data. The light distribution also shows no overdensity in the region under consideration.

\section{Analysis 2:}

For the second analysis, we used SExtractor to create a catalogue of all objects in the $I$-band image which had at least three pixels with flux greater than the $1 \sigma$ sky level noise. This catalogue, which contained mostly noise peaks, was then analysed by convolving each object in the image with progressively larger Mexican-hat filters until a maximum in signal-to-noise $v$, for the filtered object was found. The background galaxy catalogue was then selected from objects with SExtractor isophotal magnitude $I>22, v>10$, having a Mexican-hat filter radius at maximum $v$ which was larger than that measured for stars, and not having a neighboring object within 2 '.5. The resulting catalogue contained 1838 objects,

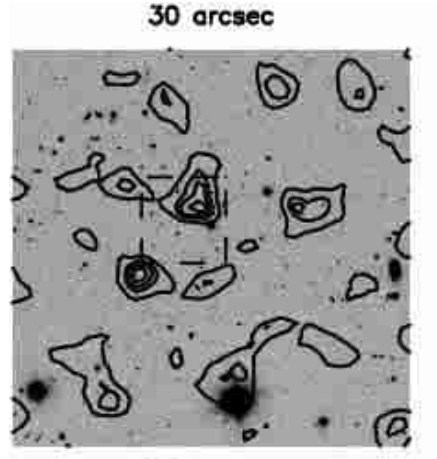

90 arcsec
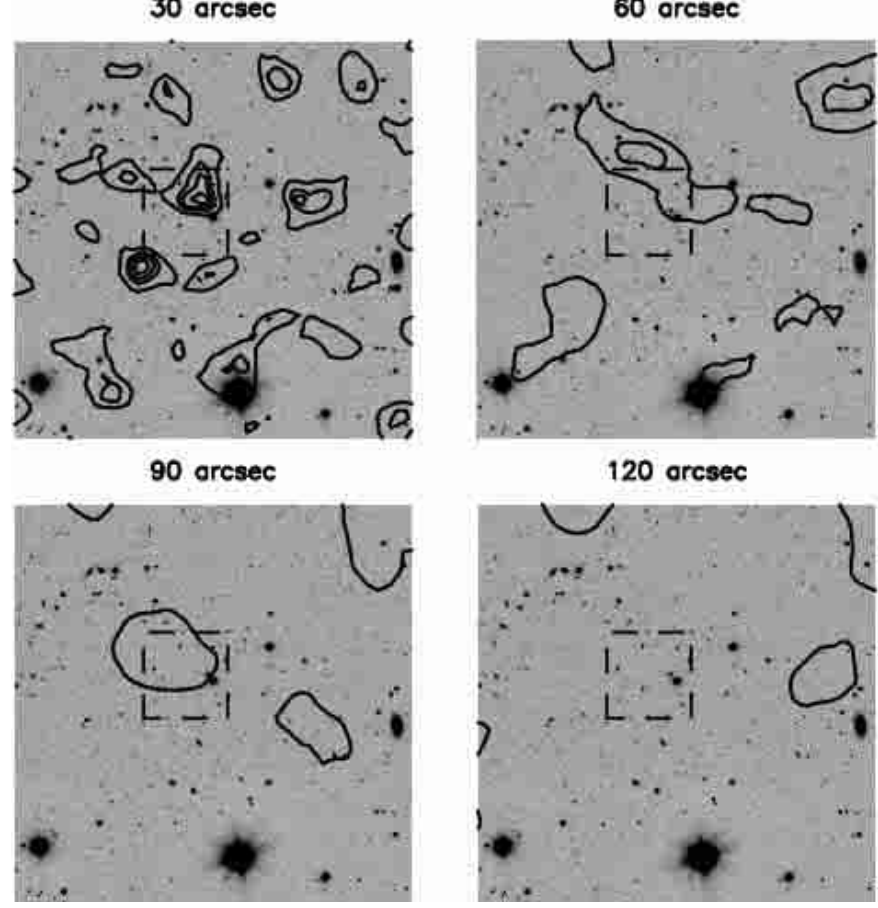

120 orcsec

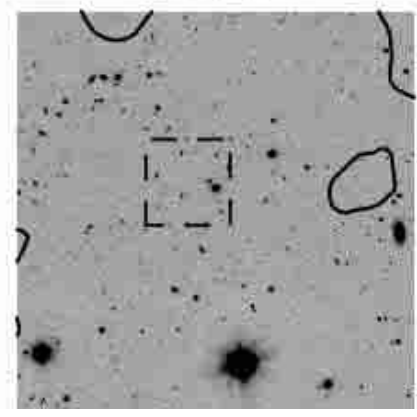

Fig. 5. Shown are $M_{\text {ap }}$ significance contours for different filter scales indicated by the labels. Contours show the levels of 1.0, 2.0, 2.5 and 3.0 $\sigma$. Dashed rectangles mark the original STIS observation. We note that the $3 \sigma$ peak at the position of our lens candidate at the smallest filter scale vanishes when the filter size is increased.

with 954 in common with the catalogue from the first analysis. Objects were detected as faint as $I=27$, although the completeness limit, as judged by where the number counts depart from a powerlaw, is at $I \sim 24.8$.

The background galaxy ellipticities were also corrected for PSF smearing to obtain shear estimates using the KSB formalism, but the methods used to reduce the noise in the shear and smear polarizabilities were different from those employed in the first analysis. The method was that given in Clowe \& Schneider (2001), using fifth-order two-dimensional polynomials to fit the stellar ellipticity and shear and smear polarizabilites as a function of position in the image. A direct comparison of the catalogues for the two analyses results in the same mean shear across the objects and an rms shear difference of 0.16 for the objects in common, while the rms shear of the objects is about 0.4 .

A mass reconstruction from direct Fourier transform of the shear field (Kaiser \& Squires 1993) is shown in Fig. 6. The large peak in the NNW portion of the image is signficant at $2.5 \sigma$, while all of the other peaks are less than $2 \sigma$. There is a detected overdensity in the vicinity of the dark lens candidate, but at only a $1 \sigma$ significance. In the next section we analyse the implications of the current null result by taking a closer look at the galaxy populations used in this and the original analysis.

\section{Interpretation}

The original STIS data gave a striking visual impression of a strong gravitational lens manifesting itself in several highly 


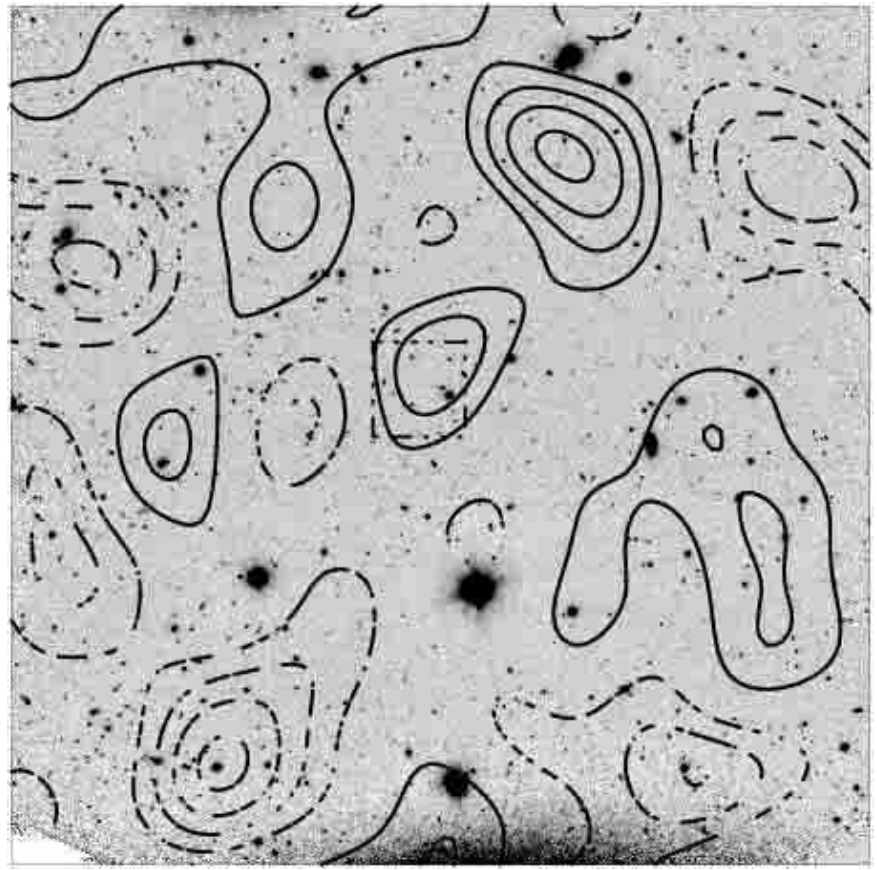

Fig. 6. The figure shows the weak lensing mass reconstruction (Kaiser \& Squires 1993) from the second analysis. The smoothing of the Map is $28^{\prime \prime}$. The highest peak (roughly on the $2.5 \sigma$ level) is close to the second peak in the North-West of the $M_{\text {ap }}$ map from the first analysis (Fig. 4). No other features above the noise level are seen in this reconstruction. Dashed contours show regions of negative $\kappa$. The contours mark $23 \%, 46 \%, 69 \%$ and $92 \%$ of the maximum and minimum peak intensity in the image respectively.

elongated arclet candidates showing a coherent alignment towards the brightest galaxy in the field (see Fig. 2 of MEH and the publicly available data under http://www.stecf. org/projects/shear/slens/slens.fits). If the features present in this field indeed originate from gravitational lensing and hence from a mass concentration of the order of a massive galaxy cluster, we should expect to detect a significant $M_{\text {ap }}$ signal with a filter scale of about $2^{\prime}$ (see Sect. 3) or a significant peak in a weak lensing mass reconstruction. Also, a larger scale overdensity in the light distribution should be detected for a massive galaxy cluster with a typical mass-to-light ratio. With the available VLT data covering the surroundings of the candidate on a field of about $7^{\prime} \times 7^{\prime}$, we fail to find both signatures of a possible massive structure at the STIS image position.

As was shown in Sect. 3, we should be able to detect either a massive galaxy cluster up to a redshift of $z \approx 0.6$ with the data at hand, or a medium-sized cluster up to a redshift of $z \approx 0.3$.

Hence, upholding the lensing hypothesis would imply that the massive structure is at substantially higher redshift that we cannot probe with our VLT data. Since we reach a significantly deeper source population with our STIS data (see also Fig. 8), we have to investigate this possibility further. In the following we try to test this hypothesis by considering common objects in the STIS and VLT analysis and their photometric properties. In the original analysis we split the detected sources in the STIS image into two samples. The first sample consists of 52 objects which represents the catalogue for our weak lensing analysis
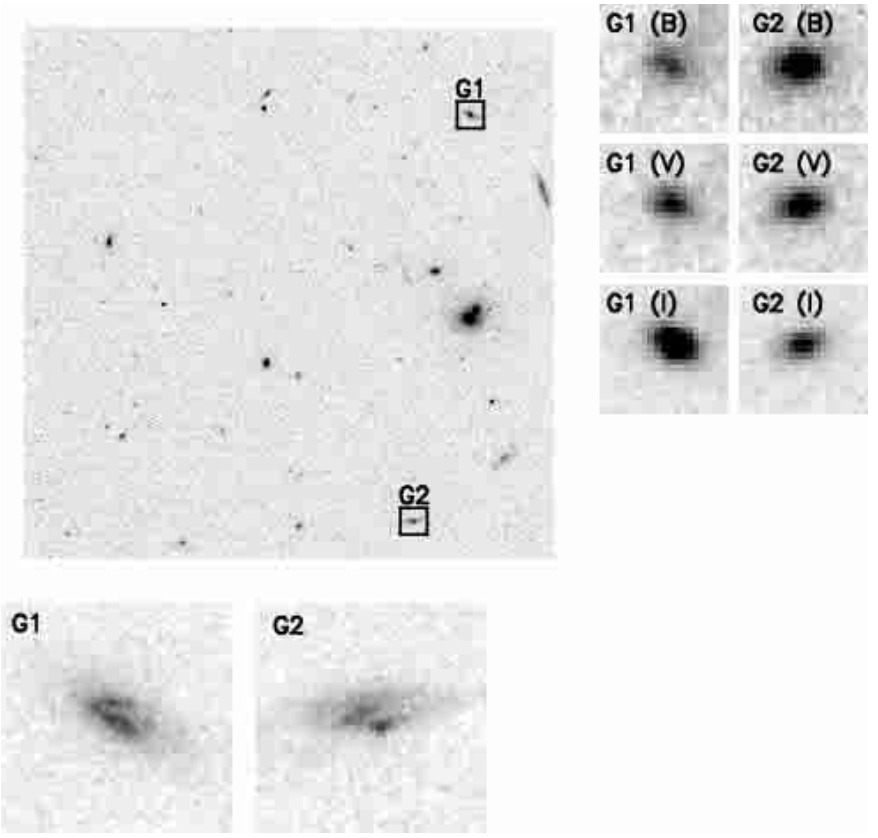

Fig. 7. We show the status of the double image candidate G1 and G2 presented in $\mathrm{MEH}$. The surface brightness for the two objects in the STIS Clear filter is nearly identical $\left(23.29 \mathrm{mag} / \mathrm{arcsec}^{2}\right.$ for G1 and $23.34 \mathrm{mag} / \operatorname{arcsec}^{2}$ for G2) and also the very similar and striking morphology suggested them to be two images of a single source. Equal parity of the images weakened the lensing hypothesis, though. Regarding the brightness in the three VLT colours (panels on the right) immediately reveals very different object colours $(B-I=3.85$ for $\mathrm{G} 1$ and $B-I=2.42$ for $\mathrm{G} 2$ ), excluding the lensing hypothesis.

using the $M_{\text {ap }}$ statistics. This analysis yields a peak at the $2.4 \sigma$ level. In the following, we regard the position of this peak as the center of our lens candidate. A second, independent object sample with 11 objects consists of all highly elongated and well resolved objects having a SExtractor axis ratio larger than 2. Most of them were rejected from the weak lensing catalogue for various reasons (for instance because of difficulties in determining an exact object center or having a too large corrected ellipticity) and we regard them as lensed arclet candidates. Considering the position angle distribution, we found that the probability for the observed tangential alignment of these objects with respect to the $M_{\text {ap }}$ center was only $0.3 \%$ when assuming a random angle distribution. In this way we ended up with two independent lensing signatures of a massive object at the $2.5 \sigma$ level. Moreover, two of these arclet candidates were considered as a multiple image candidate because of their morphological similarity and nearly identical surface brightness in the STIS Clear filter. On these issues, our VLT data shed new, important light.

First, they reveal that the two objects of our double image candidate (G1 and G2 of Fig. 2 in MEH) are different sources and cannot be due to a single, lensed object because of their colours. Object $\mathrm{G} 1$ has $B-V=1.53$ and $V-I=2.32$, while $\mathrm{G} 2$ has $B-V=1.12$ and $V-I=1.30$. Figure 7 summarises the situation for these two objects.

Next, we investigate in more detail whether the candidate arclets can still be considered as such. If their ellipticities are indeed caused by a massive structure at high redshift $(z \geq 0.6)$ 
Table 1. Given are the photometric redshift intervals (estimated with hyperz) at the $68 \%$ confidence level using the $B, V$ and $I$ images. Peaks in the probability distribution are also given. We determine only regions so that the absolute $I$ magnitudes of objects lie within $M_{*}-2<M<M_{*}+3$ as explained in the text. We consider the original strong lensed arclet candidates (G1, G2 and A1-A8), the brightest galaxy in the STIS field (BG) and 5 additional objects (V1-V5) common to the STIS and VLT weak lensing analysis. See also Fig. 8.

\begin{tabular}{|c|c|c|c|c|}
\hline object & $I-\mathrm{mag}$ & permitted redshift range & peaks & comments \\
\hline G1 & 21.74 & $0.45<z<0.75$ & $0.48 ; 0.67$ & \\
\hline \multirow[t]{2}{*}{ G2 } & 22.54 & $0.3<z<0.5$ & $0.34 ; 0.45$ & \\
\hline & & $1.62<z<1.66$ & 1.64 & \\
\hline A1 & 21.44 & $0.49<z<0.75$ & $0.54 ; 0.64$ & \\
\hline \multirow[t]{2}{*}{ A2 } & 21.55 & $0.34<z<0.415$ & 0.35 & two resolved objects \\
\hline & & $0.48<z<0.52$ & 0.51 & in STIS \\
\hline $\mathbf{A 3}$ & - & - & - & merged with a star in VLT data \\
\hline A4 & 24.34 & $0.5<z<1.6$ & $0.59 ; 0.82 ; 1.37$ & \\
\hline A5 & 25.10 & $1.49<z<1.79$ & 1.64 & \\
\hline A6 & 24.37 & $1.49<z<1.75$ & 1.55 & \\
\hline A7 & - & - & - & not detected in VLT images \\
\hline A8 & 25.05 & $1.6<z<2.7$ & - & multiple peaks \\
\hline BG & 19.09 & $0.29<z<0.43$ & 0.35 & \\
\hline \multirow[t]{2}{*}{ V1 } & 23.20 & $0.45<z<0.58$ & 0.5 & \\
\hline & & $1.32<z<1.98$ & 1.48 & \\
\hline \multirow[t]{2}{*}{$\mathbf{V} 2$} & 22.80 & $0.57<z<1.01$ & $0.71 ; 0.86 ; 0.98$ & \\
\hline & & $1.28<z<1.45$ & $1.30 ; 1.41$ & \\
\hline V3 & 21.66 & $0.42<z<0.56$ & $0.44 ; 0.54$ & \\
\hline \multirow[t]{2}{*}{ V4 } & 23.32 & $0.42<z<0.46$ & 0.45 & \\
\hline & & $1.64<z<1.69$ & 1.67 & \\
\hline V5 & 23.86 & $1.83<z<1.87$ & 1.85 & $\begin{array}{l}\text { marginal solutions } \\
\text { at } z \sim 0.3 \text { and } z \sim 3\end{array}$ \\
\hline
\end{tabular}

they must be located at even substantially higher redshift. The three bands in hand do not permit the estimate of accurate photometric redshifts (Bolzonella et al. 2000) for these objects, but do permit us to test whether high redshifts (larger than $z \approx 1$ ) would be compatible with their colours. Adding a prior about the luminosity function of the galaxies helps to constrain the permitted redshift ranges with only three bands (see e.g. Benitez 2000). We choose to consider only solutions for photometric redshifts which are compatible with an absolute magnitude range $M_{*}-2<M<M_{*}+3$ for the $I$-band. This band is rather insensitive to evolution effects since it probes the old stellar population, and we choose to use the local $M_{*}=-21.26$ (Blanton et al. 2000) deduced from the SDSS early data release. In Table 1 we present our deduced redshift constraints for the original arclet candidates (objects G1, G2 and A1-A8 in Fig. 2 of $\mathrm{MEH})$. We note that the colours of the brightest and most prominent candidates (G1, G2, A1, A2 and A4) are compatible mostly with redshifts considerably lower than unity. All the other faint objects in the sample have indeed a high probability of being placed at $z \geq 1.4$.

However, with the most prominent arclet candidates being probably at low redshift and without the multiple image candidate, the strong lensing hypothesis for these fainter sources by a high-redshift cluster looses much of its strength.

Finally, we take a closer look at the original weak lensing signal of our STIS field. The $2.4 \sigma M_{\text {ap }}$ signal has been very robust against the analysis of various subsamples of the
52 objects. We still could recover peaks close to the original signal when removing randomly either $30 \%$ and $50 \%$ of the sources or repeating the procedure with the most elliptical sources. Figure 8 shows that only 5 of these objects that are also amongst the brightest in the whole STIS field are also used in the first VLT analysis (not considering the arclet candidates). Considering the allowed redshift ranges as we did with the arclet candidates (see Table 1) indicates that these sources probably lie at low redshift. These sources lie at the faint end of the VLT sources but only at the bright end of the STIS galaxy population used in the initial analysis. Hence, as we are not able to use most of the original, faint STIS galaxy population in our current weak lensing analysis, no conclusive answers on the origin of the initial weak lensing signal can be given at this stage.

\section{Conclusions and outlook}

In $\mathrm{MEH}$ we reported on the conspicious tangential alignment of 63 galaxies in a $52^{\prime \prime} \times 52^{\prime \prime}$ high resolution STIS field. The maximum of the weak lensing $M_{\text {ap }}$ signal of 52 objects defined a formal mass peak close to the brightest elliptical galaxy in the field. Eleven other independent and elongated objects show a coherent tangential alignment towards the center defined by $M_{\text {ap }}$. In addition, two of those objects have very similar morphologies and surface brightnesses in the original STIS data suggesting a multiple lensed image. Hence, the discovery of a new, massive galaxy cluster acting as a strong 


\section{STIS}

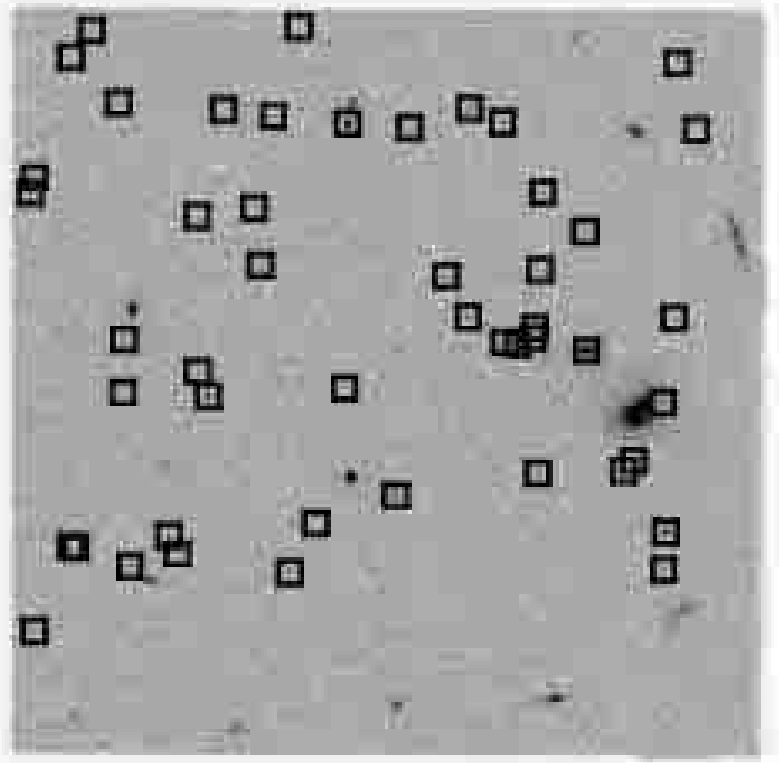

1 orcmin

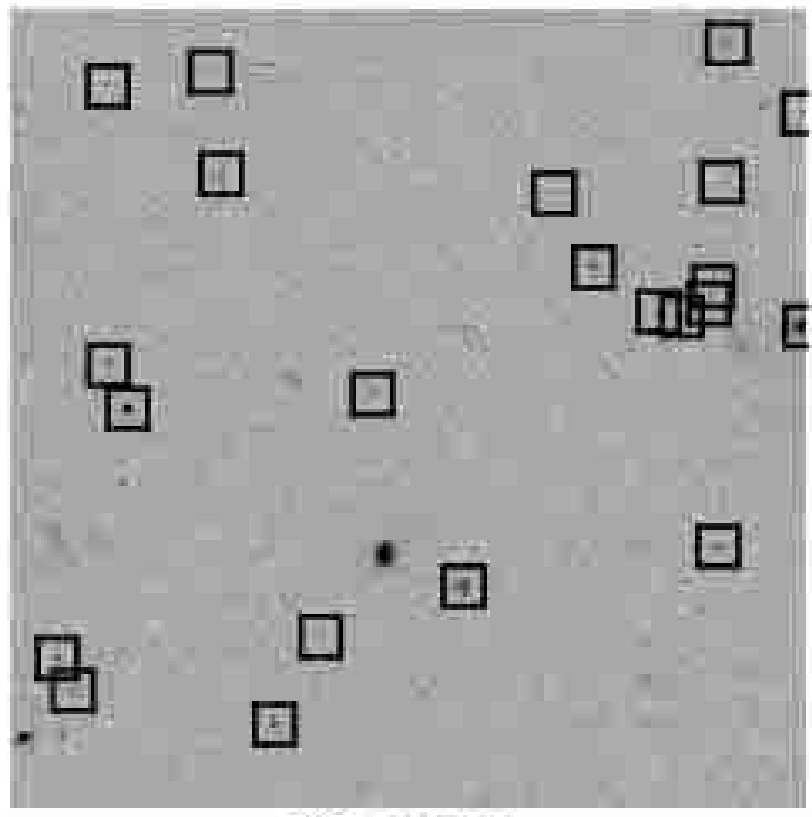

30 arcsec
VLT
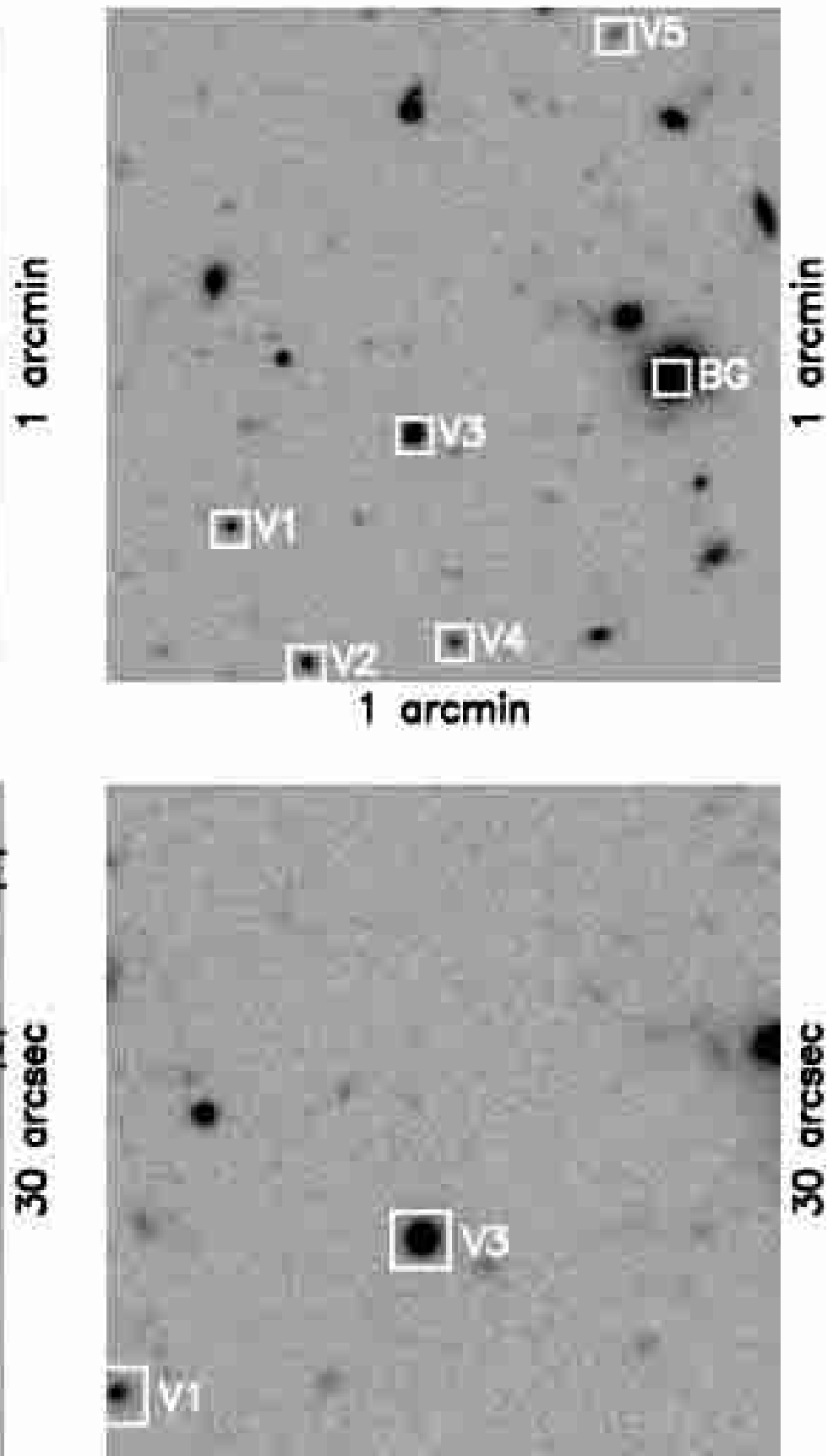

30 orcsec

Fig. 8. Shown are common objects in the original STIS and the current VLT weak lensing work (white boxes in the upper right panel; the plot does not include common objects that were amongst the STIS strong lensed arclet candidates), objects that were used in the STIS analysis only (black boxes in the upper left panel). The lower panels are 30" zooms from the upper panels. We see that many galaxies that were used in the HST/STIS analysis are still visible but too faint or too small to allow a reliable shape measurement in our high-quality VLT observations.

gravitational lens had to be taken seriously into account and to be further investigated with optical follow-up observations covering a larger field-of-view. With new, high-quality VLT data in three bands covering $7^{\prime} \times 7^{\prime}$ around the original cluster candidate, we now arrive at the following conclusions:

- The light distribution in the VLT field shows that the brightest galaxy in the STIS field is an isolated early type galaxy and not part of an optically rich galaxy cluster. There is no indication for an overdensity of the light distribution in or very close to the STIS field.

- With the data at hand we should detect, with $\geq 3 \sigma$ significance, a matter concentration of about $3 \times 10^{14} M_{\odot}$ up to a redshift of $z \leq 0.6$ with weak lensing techniques. We fail to find such a lensing signature in two independent analyses. 
- We can exclude the initial double image hypothesis for two of the very elongated objects we regarded as strongly lensed arclet candidates. Moreover, the colours of the brightest and most prominent of these candidates are only compatible with redshifts up to about 0.7 . This makes it impossible that their large elongations originate from lensing by a high-redshift cluster.

Regarding the origin of the original weak lensing signal in the STIS data, no progress could be made with the new VLT data at hand. Besides the brighter arclet candidates, only 5 additional objects are used in common in the original STIS and the new VLT weak lensing analysis. Those represent the faint end of the VLT but only the bright end in the STIS galaxy population. The remaining 47 STIS sources are too small and too faint in the high-quality VLT images to be usable for a weak lensing study. Hence, we cannot exclude that those sources represent a high redshift galaxy population and their very robust alignment is indeed caused by lensing by a mass concentration at high redshift.

With the negative conclusions regarding the initial strong lensing arguments, especially the loss of the double image candidate, a chance alignment of 52 objects on a $52^{\prime \prime} \times 52^{\prime \prime}$ field causing a signal on the $2.5 \sigma$ level is the most plausible explanation for the initial signal at this point.

The past has shown that deep HST exposures are likely to reveal highly elongated and well resolved galaxies with lenslike morphologies. Also, for the Hubble Deep Field North, a possible strong lensing configuration has been found (Hogg et al. 1996) but the lensing hypothesis has later been falsified with Keck spectroscopy (Zeff et al. 1997). Most of these stronglens candidates found in HST data can be resolved by follow-up observations on ground-based facilities, either by spectroscopy or multi-colour observations.

In contrast to clarifying these strong lens candidates, it is more difficult to confirm or discard potential discoveries of mass concentrations found with weak lensing techniques on HST images on small angular scales, as in the current case, or that presented in Umetsu \& Futamase (2000). The small fieldof-view and limited number of galaxies increase the probability for chance-alignments compared with wide-field ground-based observations. On the other hand, as was shown by this and previous studies (see e.g. Hoekstra et al. 2000), current state of the art ground based facilities are not able to probe the faint galaxy population of deep HST exposures with a resolution sufficient for weak lensing studies. Currently, only new space-based observations with ACS, having a large enough field-of-view to probe a sufficiently large area around these candidates, could finally close these cases.
Acknowledgements. We thank the ESO Director General, C. Cesarsky for approval of the DDT proposal and the service observing team at Paranal for providing us with this excellent data set. We are grateful to L. King and to the referee, J. D. Rhodes for a careful reading of the manuscript. This work was supported by the German Ministry for Science and Education (BMBF) through the DLR under the project 50 OR 0106, by the German Ministry for Science and Education (BMBF) through DESY under the project 05AE2PDA/8, and by the Deutsche Forschungsgemeinschaft (DFG) under the project SCHN 342/3-1.

\section{References}

Bartelmann, M. 1996, A\&A, 313, 697

Bartelmann, M., \& Schneider, P. 2001, Phys. Rep., 340, 291

Benitez, N. 2000, ApJ, 536, 571

Bertin, E., \& Arnouts, S. 1996, A\&AS, 117, 393

Blanton, M., Dalcanton, J., Eisenstein, D., et al. 2000, AJ, 121, 2358

Bolzonella, M., Miralles, J. M., \& Pelló, R. 2000, A\&A, 363, 476

Brainerd, T., Blandford, R. D., \& Smail, I. 1996, ApJ, 466, 623

Clowe, D., Luppino, G. A., Kaiser, N., \& Gioia, I. 2000, ApJ, 539, 540

Clowe, D., \& Schneider, P. 2001, A\&A, 379, 384

Dahle, H., Pedersen, K., Lilje, P. B., Maddox, S. J., \& Kaiser, N. 2003, ApJ, 591, 662

Erben, T., van Waerbeke, L., Bertin, E., Mellier, Y., \& Schneider, P. 2001, A\&A, 366, 717

Erben, T., van Waerbeke, L., Mellier, Y., et al. 2000, A\&A, 355, 23

Fruchter, A. S., \& Hook, R. N. 2002, PASP, 114, 144

Hoekstra, H., Franx, M., \& Kuijken, K. 2000, ApJ, 532, 88

Hoekstra, H., Franx, M., Kuijken, K., \& Squires, G. 1998, ApJ, 504, 636

Hogg, D. W., Blandford, R., Kundić, T., et al. 1996, ApJ, 467, L73

Kaiser, N., \& Squires, G. 1993, ApJ, 404, 441

Kaiser, N., Squires, G., \& Broadhurst, T. 1995, ApJ, 449, 460

Kruse, G., \& Schneider, P. 1999, MNRAS, 302, 821

Mellier, Y., van Waerbeke, L., Maoli, R., et al. 2000, ESO Messenger, 102,30

Miralles, J. M., Erben, T., Hämmerle, H., et al. 2002, A\&A, 388, 68

Monet, D., Bird, A., Canzian, B., et al. 1996, U.S. Naval Observatory: Washington DC

Navarro, J., Frenk, C., \& White, S. 1996, ApJ, 462, 563

Schirmer, M., Erben, T., Schneider, P., et al. 2003, A\&A, 407, 869

Schneider, P. 1996, MNRAS, 283, 837

Umetsu, K., \& Futamase, T. 2000, ApJ, 539, L5

Verde, L., Peng Oh, S., \& Jimenez, R. 2000, MNRAS, 336, 541

Weinberg, N. N., \& Kamionkowski, M. 2002, MNRAS, 337, 1269

Wittman, D., Margoniner, V. E., Tyson, J. A., et al. 2002, ApJL, submitted [astro-ph/0210120]

Wittman, D., Tyson, J. A., Margoniner, V. E., et al. 2001, ApJ, 557, 89

Zeff, S. E., Moustakas, L. A., \& Davis, M. 1997, ApJ, 474, L1 